\title{
Imprinting: Transfer of the following response after initlal attachment ${ }^{1}$
} RICHARD H. PORTER and LAURENCE J. STETTNER,
Wayne State University, Detroit, Mich. 48202

Bobwhite quail (Colinus virginianus) were imprinted to an auditory-visual stimulus complex. After the critical period, experimental Ss were exposed to the original auditory stimulus plus new visual object. Controls were exposed to the new visual object alone. In subsequent tests with the new object, experimental birds showed a significantly greater attachment than controls.

According to the classical conception of the imprinting process, certain species of birds form exclusive social attachments to stimuli (animate or inanimate) to which they are exposed during an early critical period in development. Guiton (1959) reports that after chicks learn to follow an object and to discriminate between it and other objects, "the following response ceases to be elicited by a strange moving object." There are thus "inhibitory effects of socialization on the following response to unfamiliar objects." A similar conclusion was presented by Sluckin \& Salzen (1961) who stated that with increased imprinting "the bird becomes less likely to respond to stimuli other than the experienced one or ones." In accordance with the above conclusions, it has been shown that the "critical period" during which stimulus exposure can elicit following behavior can be significantly lenghtened if the bird is deprived of visual and/or social experience after hatching (Moltz \& Stettner, 1961; Sluckin, 1962).

In a different line of experiments, an apparent interaction has been reported between "imprinting-like behavior and conditioning" (see Sluckin, 1965). James (1959), for example, has shown that chicks show approach and following directed towards an object (polythene ball) which had previously been hanging motionless alongside of a series of intermittent lights to which the chick had been imprinted. Similar studies (Klopfer, 1959; Abercrombie \& James, 1961) in which the CS (sound or motionless object) was presented along with the moving or intermittent imprinting stimulus (UCS) during the initial imprinting trials reveal similar results (i.e., the CS subsequently evokes following and/or approach responses when presented alone).

Since in the above studies the test object (CS) was always present during the initial imprinting sessions, the present authors decided to attempt to determine whether the following response, once established, could be transferred to a new stimulus object after it had been paired with components of the original imprinting stimulus complex. The present experiment is thus an attempt to have the following and approach responses directed towards a completely new stimulus which is initially presented after the critical period and after the bird has already shown evidence of being strongly attached to a different stimulus complex.

Subjects

\section{METHOD}

The Ss were Bob-white quail (Colinus virginianus) which were hatched in an electric forced-air incubator in the laboratory. The eggs had been obtained from the breeding stock of the Georgia Quail Farm, Savannah, Georgia. Newly hatched birds remained visually isolated from each other in individual wooden hatching boxes in the incubator until the initial imprinting trial. The birds were moved to an isolation brooder after the first imprinting session and were again visually isolated from each other. Food and water were available ad lib to the $\mathrm{Ss}$ in the isolation brooder.

\section{Apparatus}

See Porter \& Stettner (1968) for a detailed description of the imprinting alley. The imprinting stimulus (attachment object) for all Ss was a green rectangular box (GR) $4 \times 4 \times$ 6 in. with a speaker inside. A tape recording of an electronically produced sound (repetitive "beep" of $500 \mathrm{cps}$, $100 \mathrm{msec}$ on, $100 \mathrm{msec}$ off) was used as the auditory attachment stimulus. A second visual object, a red cylinder (RC) $2 \frac{1}{2}$ in. in diameter $x 7$ in. high with a speaker inside was used as the "test" object as discussed below.

\section{PROCEDURE}

The Ss were initially placed into the imprinting apparatus at 6 to $30 \mathrm{~h}$ post-hatch age. At this time, each $S$ was individually exposed to the attachment stimulus (GR \& "beep") for $30 \mathrm{~min}$. The following day (at least $12 \mathrm{~h}$ after the initial exposure) each bird was re-exposed to this same stimulus for a second $30 \mathrm{~min}$ period.

In each $30 \mathrm{~min}$ experimental session, the object moved for $10 \mathrm{~min}$ (at approximately $1 \mathrm{ft}$ per $3 \mathrm{sec}$ ), was stationary for $5 \mathrm{~min}$, moved again for $10 \mathrm{~min}$ and was stationary for the last $5 \mathrm{~min}$. Each session was thus broken up into four sub-periods; two $10 \mathrm{~min}$ periods with object moving and two 5 min periods in which the object was stationary. A Standard Electric Timer was activated whenever $S$ was standing within 12 in. behind or $6 \mathrm{in}$. in front of the moving object, or was within $8 \mathrm{in}$. of the stationary object.

Only birds which met the following criterion of attachment during the second imprinting session were retained in this experiment and used for further testing: (1) Ss had to accumulate a score of at least 4 min of positive response out of the $20 \mathrm{~min}$ that the object was moving; and (2) each $S$ was required to remain within 8 in. from the object for at least $2 \mathrm{~min}$ out of the $10 \mathrm{~min}$ that the object was stationary. Up until this point, both controls and experimental Ss had identical experiences and attachment criteria.

On Day 2, from $2-4 \mathrm{~h}$ after the second exposure session, the six experimental Ss were individually given two successive training sessions of $30 \mathrm{~min}$ each with both moving and stationary objects. During one of these sessions, Ss were again exposed to the original imprinting stimulus complex (GR \& "beep"). In the other session, Ss were exposed to a new object (RC) emitting the familiar "beep."

On the third day, each experimental $S$ was again placed into the alley and exposed to the "beeping" RC for a $30 \mathrm{~min}$ period. One to two hours after this second exposure to the "beeping" $\mathrm{RC}$, the experimental birds were tested for their response to the silent $\mathrm{RC}$. Seconds of positive response to both moving and stationary object were scored as explained above. This experiment is thus an attempt to establish a transfer of the attachment response to an object which had previously been paired with the original imprinting sound ("beep").

The control Ss were five birds which were originally exposed to the beeping GR and had met the same attachment criterion as the experimental Ss. At least $2 \mathrm{~h}$ after the second imprinting session with the "beeping" GR (Day 2), control Ss were individually exposed to the silent $\mathrm{RC}$ for $30 \mathrm{~min}$ (thus parallelling the time of the initial exposure of the experimental Ss to the "beeping" RC). On the following day (3), the controls were scored as described above for seconds of positive response to the silent $\mathrm{RC}$ during a $30 \mathrm{~min}$ session with both moving and stationary stimuli. 
It should be emphasized that all animals (both experimentals and controls) in this study were strong followers and had met the same criterion of attachment.

\section{RESULTS}

On the test trials, the experimental Ss had a mean following score of the moving object (silent RC) of $268 \mathrm{sec}$ which was significantly greater than the mean following score of $132 \mathrm{sec}$ for the controls $(t=2.23, \mathrm{df}=9, \mathrm{p}<.05)$. Three of the six experimental birds showed strong attachment to the silent RC and actually followed it to the criterion for attachment discussed above. None of the control Ss showed any evidence of attachment to the silent RC. It should be pointed out that a score of approximately $120 \mathrm{sec}$ (compared to the mean following score of $132 \mathrm{sec}$ for the control group) would have been obtained if the bird merely remained stationary and was scored for the time when the object moved past it.

With the stationary object, the experimental birds had a mean positive response of $121 \mathrm{sec}$ compared to $13 \mathrm{sec}$ for the control birds $(t=1.56, \mathrm{df}=9, \mathrm{p}<.10>.05)$. Two of the experimental Ss met the previously discussed criterion for attachment when tested with the stationary object (i.e., at least $2 \mathrm{~min}$ of positive response to the object during the 10 min that it was stationary). Again, none of the controls met this criterion when tested with the silent RC.

The above results indicate that once $S$ is induced to follow the calling $\mathrm{RC}$, it will continue to follow the $\mathrm{RC}$ to some extent even when the original imprinting sound is withdrawn. This phenomenon occurs even when the initial exposure to the RC occurs after the critical period and after Ss have already been "imprinted" to a different object.

\section{DISCUSSION}

The results of the present experiment raise several points of considerable theoretical significance. The existence of such transfer of the imprinting response greatly increases the possibilities that neonatal following (and other forms of filial attachment behavior) could be directed toward stimuli which are not present during the early sensitive period. Following based on such transfer would not be due to generalization, nor would it be due to any association of a response with a "conventional" reinforcing agent. The present data do, however, agree with earlier studies mentioned above in which the original imprinting stimulus is interpreted as acting analogously to an UCS, and by pairing the same with a new stimulus (CS) this new stimulus will in turn elicit approach and following responses (CR). This type of transfer may represent an important factor in the socialization process of young birds.

The existence of such a transfer effect also seems significant in that it appears to contradict traditional conceptualizations of the imprinting process, wherein the characteristics of the "parental object" can only be impressed upon the neonate by exposure during a brief critical period. If such were the case, and if following is a result of this imprinting process, then how could a new stimulus, in the post-critical period, come to elicit the same response if it was markedly dissimilar from the original stimulus? It is especially significant that this can occur even when (as in our experiment) there has been exposure to another test object (and, presumably, imprinting of that object) during the early critical period.

Imprinting, as Salzen (1966) has recently reiterated, essentially refers to a process of "goal acquisition" or attachment to an object which will serve as a focus of a variety of social behaviors, rather than to the acquisition of any specific response (such as following). However, we need a response criterion to determine whether such attachment has occurred, and current investigators favor preferences for an object (established by exposure) as evidence for imprinting in neonates (Sluckin, 1965; Klopfer, 1967). Preference, however, has also been shown (Sluckin, 1962; Asdourian, 1967) to be determined in some cases by post critical period exposure, so that expunging the following response from imprinting does not solve the problem of post-critical period attachments. The best account of such attachments is probably provided by Salzen's (1966) neuronal model of imprinting. In this hypothesis, initial exposure establishes a familiar pattern, and any subsequent large deviations from this pattern tend to be avoided (e.g., fear response to "strange" stimuli). According to this explanation, if there is no early exposure to any type of stimulus pattern, then no familiar pattern will be set up, and attachment will still occur to the first object presented to the animal, even though this may be well past the age at which such attachments usually occur. Moreover, if after an initial attachment model has been set up, we "enforce" exposure to a new stimulus complex, then features of that complex may be incorporated into the neuronal model and become part of the familiar pattern. We might interpret the results of the present experiment in this manner.

\section{REFERENCES}

ABERCROMBIE, B. \& JAMES, H. The stability of the domestic chick's response to visual flicker. Animal Behaviour, 1961, 9, 205-212.

ASDOURIAN, D. Object attachment and the critical period. Psychonomic Science, 1967, 7, 235.

GUITON, P. Socialization and imprinting in Brown Leghorn chicks. Animal Behaviour, 1959, 7, 26-34

JAMES H. Flicker: An unconditioned stimulus for imprinting. Canadian Journal of Psychology, 1959, 13, 59-67.

KLOPFER, P. H. The development of sound-signal preferences in ducks. Wilson Bulletin, 1959, 71, 262-266.

KLOPFER, P. H. Stimulus preferences and imprinting. Science, 1967, 156, 1394-1396.

MOLTZ, H., \& STETTNER, L. J. The influence of patterned-light deprivation on the critical period for imprinting. Journal of Comparative \& Physiological Psychology, 1961, 54, 279-283.

PORTER, R. H., \& STETTNER, L. J. Visual and auditory influences on the following response of the Bob-white Quail (Colinus virginianus). Journal of Comparative and Physiological Psychology, in press.

SALZEN, E. A. In L. R. Aronson, D. S. Lehrman, J. S. Rosenblatt, \& E. Tobach (Eds.), Development and evolution of behavior. New York Freeman, 1966.

SLUCKIN, W. Perceptual and associative learning. Symposium of the Zoological Society of London, 1962, 8, 193-198.

SLUCKIN, W. Imprinting and early learning. Chicago: Aldine Pub. Co., 1965.

SLUCKIN, W., \& SALZEN, E. A. Imprinting and perceptual learning. Quarterly Journal of Experimental Psychology, 1961, 13, 65-77. NOTE

1. Supported by NSF Grant GB 1854 to Laurence J. Stettner and NSF Research Participation Fellowship to Richard H. Porter. 